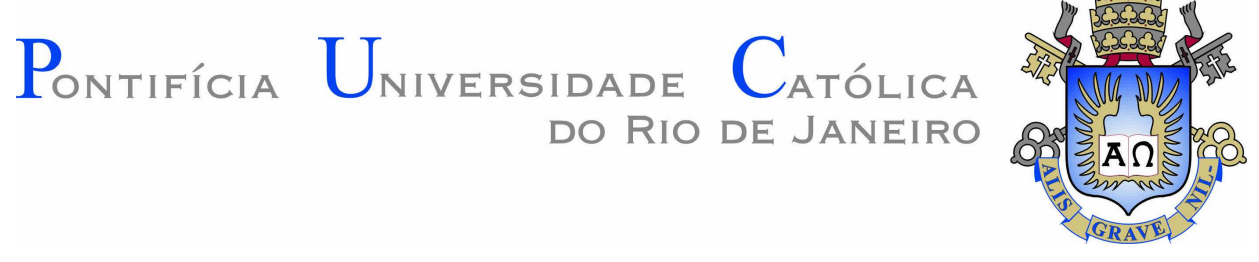

Julia Gomes Ázara de Oliveira

\title{
Microscopia Digital e Análise de Imagens para Caracterização de Tubos Compósitos Fabricados por Enrolamento Filamentar
}

Dissertação de Mestrado

Dissertação apresentada como requisito parcial para obtenção do título de Mestre em Engenharia Metalúrgica e de Materiais pelo Programa de PósGraduação em Engenharia Metalúrgica do Departamento de Ciência dos Materiais e Metalurgia da PUC-Rio.

Orientadores: José Roberto Moraes d'Almeida Sidnei Paciornik

Rio de Janeiro

Abril de 2008 


\section{Pontifícia Universidade Católica $_{\text {a }}$ \\ DO RIO DE JANEIRO}

Julia Gomes Ázara de Oliveira

\section{Microscopia Digital e Análise de Imagens para Caracterização de Tubos Compósitos Fabricados por Enrolamento Filamentar}

Dissertação apresentada como requisito parcial para obtenção do grau de Mestre em Engenharia Metalúrgica e de Materiais pelo Programa de Pós-Graduação em Engenharia Metalúrgica do Departamento de Ciência dos Materiais e Metalurgia da PUC-Rio. Aprovada pela Comissão Examinadora abaixo assinada.

Sidnei Paciornik

Orientador

Departamento de Engenharia de Materiais e Metalurgia - PUC-Rio

José Roberto Moraes d'Almeida

Co-orientador

Departamento de Engenharia de Materiais e Metalurgia - PUC-Rio

Marcos Henrique de Pinho Mauricio

Departamento de Engenharia de Materiais e Metalurgia - PUC-Rio

Verônica Maria de Araújo Calado

Escola de Química - UFRJ

José Eugenio Leal

Coordenador(a) Setorial do Centro Técnico Científico - PUC-Rio 
Todos os direitos reservados. É proibida a reprodução total ou parcial sem autorização da Universidade, da autora e dos orientadores.

\section{Júlia Gomes Ázara de Oliveira}

Graduou-se em Engenharia Mecânica na UERJ (Universidade do Estado do Rio de Janeiro) em 2004. É Engenheira de Equipamentos da PETROBRAS, trabalhando na Gerência de Engenharia Submarina da Engenharia de Produção do E\&P.

Ficha Catalográfica

Oliveira, Julia Gomes Ázara de

Microscopia digital e análise de imagens para caracterização de tubos compósitos fabricados por enrolamento filamentar / Julia Gomes Ázara de Oliveira ; orientadores: Sidnei Paciornik José Roberto Moraes d'Almeida. - 2008.

82 f. : il. ; $30 \mathrm{~cm}$

Dissertação (Mestrado em Ciência dos Materiais e Metalurgia)-Pontifícia Universidade Católica do Rio de Janeiro, Rio de Janeiro, 2008.

Inclui bibliografia

1. Ciência dos materiais e metalurgia - Teses. 2. Enrolamento filamentar. 3. Material compósito. 4. Microscopia digital. 5. Análise de imagem. I. Paciornik, Sidnei. II. Almeida, José Roberto Moraes d'. III. Pontifícia Universidade Católica do Rio de Janeiro. Departamento de Ciência dos Materiais e Metalurgia. IV. Título. 


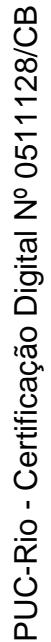

Para meus pais, Ivo e Vera, pelo amor incondicional. 


\section{Agradecimentos}

A Deus, por Sua infinita bondade.

À minha família, pelo amor, respeito e cuidado sempre demonstrados.

Aos meus orientadores, Prof. José Roberto Moraes d'Almeida e Prof. Sidnei Paciornik, que não somente por palavras, mas também pelo grande exemplo de profissionais e pessoas que são, sempre me incentivaram.

Ao apoio e colaboração do Marcos Henrique de Pinho Maurício, que com muita paciência e competência, foi peça fundamental para a montagem de nosso primeiro mosaico no MEV.

À professora Verônica Calado, pelo tempo dedicado e pela utilização do laboratório da Escola de Química da UFRJ.

Aos colegas José Frederico Fonseca Assis de Almeida e Eduardo Dumans, pelos excelentes trabalhos realizados que muito contribuíram para o desenvolvimento da minha dissertação.

Aos professores e funcionários do departamento, pelos ensinamentos e pela ajuda.

Aos meus amigos, pelo carinho e incentivo em momentos difíceis.

À Petrobras, pelo incentivo à finalização deste trabalho.

Ao CNPq, à CAPES e à PUC-Rio, pelos auxílios concedidos. 


\section{Resumo}

Oliveira, Júlia Gomes Ázara; d'Almeida, José Roberto Moraes e Paciornik, Sidnei. Microscopia Digital e Análise de Imagens para Caracterização de Tubos Compósitos Fabricados por Enrolamento Filamentar. Rio de Janeiro, 2008. 82p. Dissertação de Mestrado - Departamento de Ciência dos Materiais e Metalurgia, Pontifícia Universidade Católica do Rio de Janeiro.

Tubos de material compósito - matriz polimérica reforçada por fibra de vidro - fabricados pela técnica de enrolamento filamentar, foram caracterizados através de microscopia eletrônica digital e processamento de imagens. Três tubos foram fabricados em equipamento próprio seguindo parâmetros de enrolamento similar. Um tubo comercial fabricado por empresa especializada, com parâmetros de enrolamento mais complexos, também foi caracterizado. Para tal, seções circunferenciais foram observadas em um microscópio eletrônico de varredura com captura digital de imagem. Mosaicos de imagens foram gerados, permitindo obter informação com boa resolução local e, simultaneamente, grande abrangência espacial. Assim, foi possível realizar uma caracterização que abrangia desde o tamanho e forma de fibras individuais até a distribuição espacial de milhares de fibras em uma vasta área da amostra. Foram cridas rotinas de processamento e análise de imagens para medir dados como diâmetro, fator de forma, fração volumétrica e ângulo de enrolamento de fibras. Além disso, uma rotina específica foi desenvolvida para a identificação automática das várias camadas de fibras presentes no tubo comercial.

\section{Palavras-chave}

Enrolamento filamentar; material compósito; microscopia digital; análise de imagem. 


\section{Abstract}

Oliveira, Júlia Gomes Ázara; d'Almeida, José Roberto Moraes and Paciornik, Sidnei (Advisors). Digital Microscopy and Image Analysis for the Characterization of Filament Wound Composite Pipes - Rio de Janeiro, 2008. 82p. MSc. Dissertation - Departamento de Ciência dos Materiais e Metalurgia, Pontifícia Universidade Católica do Rio de Janeiro.

Pipes made from composite material - polymer matrix reinforced with glass fibers - manufactured by filament winding, were characterized by scanning electron microscopy and image analysis. Three pipes were manufactured with equipment owned by the research group, following similar winding parameters. A commercial tube made by a specialized company, with more complex winding conditions, was also characterized. Circumferential sections were observed in a scanning electron microscope with digital image acquisition. Image mosaics were created, providing information with good spatial resolution and, at the same time, wide spatial coverage. Thus, it was possible to characterize size and shape of individual fibers and, simultaneously, obtain the spatial distribution of thousands of fibers within a large sample area. Image processing and analysis routines were created to measure fiber diameter, shape factor, area fraction and winding angle. A specific routine was developed for the automatic identification of the several fiber layers present in the commercial pipe.

\section{Keywords}

Filament winding; composite material; digital microscopy; image analysis. 


\section{Sumário}

1 Introdução 14

1.1. Materiais Compósitos: Uma Visão Geral 14

$\begin{array}{ll}\text { 1.2. Objetivo do trabalho } & 15\end{array}$

2 Revisão da Literatura $\quad 17$

2.1. Enrolamento Filamentar 17

2.1.1. Enrolamento Molhado 18

2.1.2. Configuração do Enrolamento 20

2.1.2.1. Enrolamento Circunferencial 21

2.1.2.2. Enrolamento Helicoidal 21

2.1.2.3. Enrolamento Polar 23

2.1.3. Variáveis do processo e exemplos de aplicação 24

3 Materiais e Métodos 26

3.1. Fabricação 26

3.2. Análise Microestrutural 28

3.2.1. Preparação das amostras 28

3.2.2. Processamento Digital de Imagens 30

3.2.2.1. Formação e Digitalização da Imagem 31

3.2.2.2. Pré-processamento 33

3.2.2.3. Segmentação 39

3.2.2.4. Pós-processamento 40

3.2.2.5. Extração de Atributos 43

4 Resultados e Discussão $\quad 45$

4.1. Tubos Fabricação Própria 45

4.1.1. Resultados 45

4.1.1.1. Tubo $1 \quad 45$

4.1.1.2. Tubo $2 \quad 46$ 
4.1.1.3. Tubo $3 \quad 49$

4.1.2. Discussão 51

4.2. Tubo Comercial 55

4.2.1. Primeira etapa: Preparação da Imagem 56

4.2.2. Segunda Etapa: Discriminação das Camadas 63

$\begin{array}{ll}\text { 4.2.3. Resultados } & 70\end{array}$

4.2.4. Discussão 72

$\begin{array}{ll}\text { 4.2.4.1. Considerações Complementares } & 73\end{array}$

5 Conclusões e Sugestões para Trabalhos Futuros 74

5.1. Conclusões 74

5.2. Sugestões para Trabalhos Futuros 75

6 Referências Bibliográficas $\quad 76$

$\begin{array}{ll}7 \text { Apêndice I } & 79\end{array}$ 


\section{Lista de figuras}

Figura 1 - Ilustração de um equipamento de Enrolamento Filamentar [12]. 19

Figura 2 - Elementos sendo fabricados por Enrolamento Filamentar. 20

Figura 3 - Enrolamento circunferencial [14]. 21

Figura 4 - Enrolamento helicoidal [14]. 22

Figura 6 - Enrolamento polar [16] 23

Figura 7 - Equipamento utilizado para a fabricação dos tubos. 26

Figura 8 - Tubo fabricado no equipamento de Enrolamento Filamentar $\begin{array}{ll}\text { apresentado na Figura } 7 . & 28\end{array}$

Figura 9 - Representação esquemática do corte circunferencial. 28

Figura 10 - Etapas do processamento digital de imagens. 30

Figura 11 - Imagem capturada no microscópio óptico. 31

Figura 12 - Imagens capturadas no MEV. 32

Figura 13 - Mosaico 3x10 obtido para a amostra 1 (amostra do tubo 1).

Aumento: 50X. 34

Figura 14 - Mosaico 4x9 obtido amostra 2A (amostra do tubo 2).

Aumento: 50X. 35

Figura 15 - Imagem com detalhe do mosaico. 36

Figura 16 - Esquema de uma operação local, onde os pixels mais escuros, centrais em ambos os kernels $3 \times 3$, recebem na imagem resultante, um valor calculado a partir do valor de seus vizinhos, representados em tons mais claros [21].

Figura 17 - Aplicação do Filtro Sigma para a redução do ruído. (a) Imagem de entrada (apresentada na Figura 15) (b) Imagem resultante. 38

Figura 18 - Histograma da Figura 17(b). 40

Figura 19 - Resultado da segmentação automática. $\quad 40$

Figura 20 - Detalhe de uma região do mosaico onde é possível observar "vazios" nas fibras. 41

Figura 21 - Resultado do preenchimento dos "vazios" das fibras. 42

Figura 22 - Imagem após a aplicação do algoritmo do Divisor de Águas, 
utilizado para estabelecer as fronteiras entre as fibras.

Figura 23 - Três geometrias e seus respectivos fatores de forma

Figura 24 - Porcentagem de área de cada classe de fator de forma em relação

à área total das fibras da amostra. Amostra 1.

Figura 25 - Diâmetro médio de cada classe de fator de forma. Amostra 1.

Figura 26 - Porcentagem de área de cada classe de fator de forma em relação

à área total das fibras da amostra. Amostra 2A.

Figura 27 - Diâmetro médio de cada classe de fator de forma. Amostra 2A.

Figura 28 - Porcentagem de área de cada classe de fator de forma em relação à área total das fibras da amostra. Amostra 2B.

Figura 29 - Diâmetro médio de cada classe de fator de forma. Amostra 2B. 48

Figura 30 - Porcentagem de área de cada classe de fator de forma em relação à área total das fibras da amostra. Amostra 3A.

Figura 31 - Diâmetro médio de cada classe de fator de forma. Amostra 3A. $\quad 50$

Figura 32 - Porcentagem de área de cada classe de fator de forma em relação

à área total das fibras da amostra. Amostra 3B.

Figura 33 - Diâmetro médio de cada classe de fator de forma. Amostra 3B. 51

Figura 34 - Fração da área total de fibras para cada amostra. 52

Figura 35 - Detalhe do mosaico da Amostra 1. 53

Figura 36 - Detalhe do mosaico da Amostra 2 A. 53

Figura 37 - Detalhe do mosaico da Amostra 2B. 54

Figura 38 - Detalhe do mosaico da Amostra 3 A. 54

Figura 39 - Detalhe do mosaico da Amostra 3B. 55

Figura 40 - Mosaico obtido pela concatenação das 28 imagens capturadas no MEV.

Figura 41 - Seqüência para equalização da iluminação das imagens do mosaico. 58

Figura 42 - Mosaico com iluminação corrigida. 59

Figura 43 - Mosaico após a segmentação. $\quad 60$

Figura 44 - Seqüência para preenchimento dos "vazios" das fibras. 61

Figura 45 - Fronteiras geradas pelo algoritmo do Divisor de Águas. 62

Figura 46 - Mosaico após a geração de fronteiras pelo algoritmo do Divisor de Águas. $\quad 62$

Figura 47 - Seqüência para discriminação das camadas. $\quad 64$ 
Figura 48 - Esquema ilustrativo da definição de "calibre".

Figura 49 - Mosaico após a eliminação das camadas com objetos mais elípticos. 66

Figura 50 - Mosaicos após a aplicação de várias dilatações.

Figura 51 - Mosaico após a eliminação por tamanho dos objetos remanescentes das camadas excluídas. 67

Figura 52 - Mosaico após a eliminação de 3 dos 4 grandes objetos. 68

Figura 53 - (a) Mosaico com apenas duas camadas. (b) Detalhe da região das camadas.

Figura 54 - Mosaico com a primeira camada isolada. $\quad 69$

Figura 55 - Mosaico com a segunda camada isolada. 70

Figura 56 - Apresentação, em cores, das camadas de enrolamento identificadas. 71

Figura 57 - Mosaico 4x9 da Amostra 2B. 80

Figura 58 - Mosaico 4x9 da Amostra 3A. Aumento: 200X. 81

Figura 59 - Mosaico 4x9 da Amostra 3B. Aumento: 200X 82 


\section{Lista de tabelas}

Tabela 1 - Nomenclaturas das amostras. $\quad 29$

Tabela 2 - Etapas de lixamento e polimento. 29

Tabela 3 - Porcentagem de área de cada classe de fator de forma em relação à área total das fibras da amostra e os respectivos diâmetros médios. Amostra 1.

Tabela 4 - Porcentagem de área de cada classe de fator de forma em relação à área total das fibras da amostra e os respectivos diâmetros médios. Amostra $2 \mathrm{~A}$.

Tabela 5 - Porcentagem de área de cada classe de fator de forma em relação à área total das fibras da amostra e os respectivos diâmetros médios. Amostra 2B.

Tabela 6 - Porcentagem de área de cada classe de fator de forma em relação à área total das fibras da amostra e os respectivos diâmetros médios. Amostra 3A.

Tabela 7 - Porcentagem de área de cada classe de fator de forma em relação à área total das fibras da amostra e os respectivos diâmetros médios. Amostra 3B.

Tabela 8 - Fração da área total de fibras para cada amostra.

Tabela 9 - Diâmetros e ângulos de enrolamento médios das camadas identificadas. 